\title{
ESTUDio DEL SISTEMA PARA LA GESTIÓN DE INTERRUPCIONES OMS, EN REDES DE DISTRIBUCIÓN ELÉCTRICA Y DE LOS REQUERIMIENTOS PARA SU IMPLEMENTACIÓN
}

\author{
Washington Benalcázar ${ }^{1, *}$, Roberto C. Robalino ${ }^{2}$ y Santiago Espinosa ${ }^{3}$
}

\section{Resumen}

El Sistema de Gestión de Interrupciones (Outage management System - OMS) forma parte de la red inteligente en donde su sistema de información orientado a asistir al operador del centro de control del Sistema de Servicio de Energía Eléctrica (SDEE) en las labores relacionadas con la gestión de interrupciones, es decir, la localización de interrupciones y la restauración del servicio. Las funciones principales son determinar la localización de las interrupciones y proponer la estrategia de restauración más conveniente, logrando mantener una continuidad en el servicio a los usuarios. Además, debe suministrar estos datos al Sistema de Comunicación Informático, para mantener informado, tanto a los usuarios afectados por las interrupciones, como a los operadores que realizarán proceso de restablecimiento del servicio.

Palabras clave: Sistema de Gestión de Clientes CIS, Sistema de Gestión de Fallas - DMS, Sistema de Información Geográfica - GIS, Supervisión, Control y Adquisición de Datos - SCADA.

\section{Abstract}

The System of Management Interruptions (Outage Management System - OMS) is part of the smart grid where its information system designed to assist the operator in the control center of the System Electric Service (SDEE) in the work - related interrupt handling, which means, the location of outage sand service restoration. The principal functions are determining the localization of the interruptions and proposing the most convenient strategy to restore service, while maintaining continuity in the service to the users. Also it must provide this data to the System of Communication Information to maintain information, for both the users affected by the interruptions, as well as the operators who work to reestablish the service.

Keywords: Distribution Management System DMS, Customer Information System CIS, Geographic information System GIS, Supervisory Control And Data Acquisition SCADA.

\footnotetext{
$\overline{1, *}$ Estudiante de Ingeniería Eléctrica, Universidad Politécnica Salesiana. Autor para correspondencia washbenal@ hotmail.com

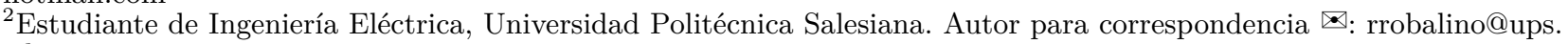
edu.ec

${ }^{3}$ Docente de la Carrera de Ingeniería Eléctrica, Universidad Politécnica Salesiana. Autor para correspondencia sespinosa@ups.edu.ec
}

Recibido: 16-04-2013, Aprobado tras revisión: 10-06-2013.

Forma sugerida de citación: Benalcázar, W.; Robalino, R. y Espinosa, S. (2013). "Estudio del sistema para la gestión de interrupciones OMS, en redes de distribución eléctrica y de los requerimientos para su implementación". INGENIUS. N. ${ }^{\circ}$ 9, (Enero-Junio). pp. 42-49. ISSN: 1390-650X. 


\section{Introducción}

Los organismos reguladores y clientes continúan aumentando en el mercado eléctrico ecuatoriano sus exigencias respecto a la calidad del suministro de energía, para cumplir con estas demandas y restricciones, las empresas distribuidoras están dirigiéndose hacia la implementación de estrategias más eficientes de gestión de interrupción, de operación y control de la red eléctrica.

En este contexto, se considera importante para el desarrollo y la sostenibilidad de energía a nivel mundial, el estudio sobre la red eléctrica inteligente (Smart Grid), misma que se ha convertido en la tendencia de la industria de la electricidad que apunta a su futura implementación y desarrollo.

\subsection{Definición y estructura del OMS [1]}

Es un sistema de información orientado a asistir al operador del centro de control, denominado Sistema de Servicio de Energía Eléctrica (SDEE), este se encarga de las labores relacionadas con la gestión de interrupciones, lo que significa, localización de interrupciones y la restauración del servicio.

\subsection{Definición y descripción de las funciones del negocio del OMS}

Entre las funciones de negocio más relevantes, en relación con las operaciones se tiene las siguientes: localización de falla, aislamiento y restauración del servicio (FLIR), la automatización avanzada de la distribución (ADA), y el análisis y modelado de la operación de distribución (DOMA), que se detalla a través del proyecto llamado Intelligrid de Instituto de Investigación de Energía Eléctrica $\left(\mathrm{EPRI}^{1}\right)$ de EE. UU.

\subsection{EPRI - INTELLIGRID ${ }^{2}$}

El Instituto de Investigación de Energía Eléctrica (EPRI), creado para la investigación, de carácter independiente, de interés público, trabaja en fomentar una base técnica para una red eléctrica inteligente en donde las conexiones eléctricas se relacionen con las comunicaciones y el control por ordenador para conseguir enormes beneficios en cuanto a fiabilidad, capacidad y mejorar el servicio a los usuarios.

EPRI destaca que el objetivo antes citado se alcanzará a través de un proyecto llamado IntelliGrid. La idea básica con IntelliGrid es que su arquitectura se compone de transmisión automática de datos a dispositivos, personas y sistemas, bajo un marco normativo común para integrar los sistemas de energía y la comunicación y así mejorar la calidad y la seguridad de la electricidad.

Este proyecto proporcionará utilidades con la metodología, las herramientas y recomendaciones para estándares y tecnologías en los sistemas de aplicación, tales como medición avanzada, automatización en la distribución, y respuesta a la demanda.

\subsection{Localización de falla, aislamiento y restau- ración del servicio $\left(\right.$ FLIR $\left.^{3}\right)$}

Esta aplicación detecta la falla, determina la sección de falla y la probable ubicación de la misma y recomienda un aislamiento óptimo de los lugares con fallas del alimentador de distribución y los procedimientos para el restablecimiento de los servicios a los lugares que no han tenido problema.

\subsubsection{Localización de fallas}

Esta subfunción se inicia con los datos de entrada del SCADA, tales como bloqueos, las indicaciones de fallos y por las aportaciones del OMS, se determina el instrumento de protección, que ha despejado la falla, y así se ubica el lugar exacto donde se encuentra desenergizado y evalúa el lugar actual o probable en la que se pueda producir la falla. Distingue fallas despejadas por instrumentos de protección de aquellos despejados por fusibles, localiza interrupciones transitorias.

\subsubsection{Análisis y modelado de la operación de la distribución (DOMA ${ }^{4}$ )}

Se basa en un flujo de energía desequilibrado en tiempo real, de modo que se van cambiando las condiciones de operación de manera dinámica. Analiza los resultados de las simulaciones del flujo de energía y proporciona al operador el resumen de este análisis, para que este tome decisiones de reconfiguración y operación. Adicionalmente, provee a otras aplicaciones con las mediciones para cada elemento del sistema de distribución, desde la subestaciones hasta los centros de carga en el secundario.

\subsection{Automatización avanzada de la distribución $\left(\mathrm{ADA}^{5}\right)$}

Tiene como finalidad mejorar la confiabilidad y la calidad de energía, para poder tener un sistema eléctrico

\footnotetext{
${ }^{1}$ EPRI por sus siglas en inglés: Electric Power Research Institute.

${ }^{2}$ INTELLIGRID: La Arquitectura IntelliGrid fue patrocinado por el Instituto de Innovación Electricidad (E2I), un miembro del Instituto de Investigación de Energía Eléctrica (EPRI) miembros de la familia. El proyecto fue IntelliGrid financiado por el Consorcio de Infraestructura Eléctrica para apoyar una Sociedad Digital (CEIDS).

${ }^{3}$ FLIR por sus siglas en inglés Fault Location, Isolation and Service Restoration

${ }^{4}$ DOMA por sus siglas en inglés Distribution Operation Modeling and Analysis

${ }^{5}$ ADA por sus siglas en inglés Advanced Distribution Automation.
} 
más eficiente, mediante la automatización de los procesos de distribución, tales como análisis y preparación de datos lo más cercano al tiempo real, optimización de la toma de decisiones y control de las operaciones de distribución.

\subsubsection{Definición y descripción de los sistemas que se relacionan al OMS [2]}

El objetivo de investigar los sistemas que se relacionan con el OMS es determinar una metodología que procese estos datos y los transforme en información útil, que puede ser utilizada por las funciones de análisis disponibles en el DMS / OMS y sirva de referencia para la toma de decisiones operativas confiables.

Para hacer frente al ambiente competitivo y emplear las nuevas tecnologías de la información, las empresas distribuidoras deben perseguir la implementación e integración de:

- Sistemas de información diseñados para la gestión de negocios de la empresa, el procesamiento de datos comerciales, atención al cliente y administración de recursos, tales como Sistema de Gestión de Negocios (Business Management System-BMS), Sistema de Información al Cliente (Customer Information System-CIS).

- Sistemas de información diseñados para la supervisión, control y análisis del funcionamiento como: SCADA, DMS, OMS. GIS, etc.

\section{Normativas de requerimientos para un OMS [3] [4] [5]}

Para que una norma pueda ser de gran utilidad, debemos tomar en cuenta la creación de procesos en el sistema de gestión de interrupciones, los cuales servirán para dar una continuidad en la energía eléctrica, procesos que han sido implementadas en varias empresas de distribución eléctricas en el mundo.

En consecuencia, la descripción de requerimientos funcionales y la terminología empleada son útiles como base al momento de crear modelos de procesos de software de las compañías eléctricas. Esto ayuda a evaluar la idoneidad de la información del sistema, es decir, el grado en que el sistema cumple los requisitos de los usuarios, como también muestra el uso de mapas de procesos para evaluar el negocio de la Tecnología de información (Informatic Technologies - TI) y regular las funciones de gestión de interrupciones.

\subsection{Requerimientos funcionales basados en los estándares IEC y EPRI}

La arquitectura común propuesta por EPRI y las funciones de negocio estandarizadas por IEC, se derivan sobre la base de teorías explícitas con respecto a los procesos de negocio para la operación óptima del sistema eléctrico.

La Ingeniería de Requerimientos fue creada con la finalidad de determinar, los requisitos funcionales, que facilitan la identificación de actividades y actores, para crear modelos de procesos; y, se utilizan para asignar las funciones del sistema a las actividades para investigar la idoneidad de interrupciones del sistema DMS.

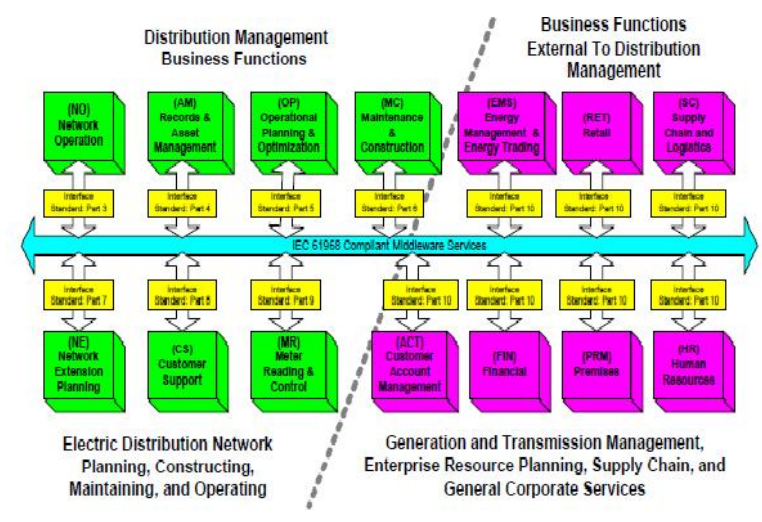

Figura 1. Funciones del negocio de la gestión de distribución [6].

De la Figura 1, se puede rescatar las funciones de mayor utilidad empleadas por el Sistema de Gestión de Interrupciones, para el manejo de grandes empresas de distribución, las cuales son:

- Operación de la red (Network Operation - N.O.) se compone de las funciones de supervisar y controlar la red eléctrica. Esto incluye también las funciones relativas a la gestión de fallos, así como estadística de la operación. Algunos ejemplos de los componentes dentro de esta función de negocio son la supervisión de la red estatal, local y el control de potencia reactiva, control de interruptor remoto, la coordinación de incidente grave, localización de fallas mediante el análisis de los detectores de fallas y / o dificultad para las llamadas, información de incidencias de clientes, análisis de funcionamiento del dispositivo, etc.

- Atención al cliente (Customer Support C.S.) consiste en el servicio al cliente y gestión de llamadas de emergencia. Esto incluye realizar reportes de situación de solicitud de servicio, el estado de trabajo, conexión a los clientes, las llamadas de interrupción, la calidad de la energía, las notificaciones previstas paradas, los medios de comunicación y la historia de interrupción. 


\section{Funciones complementarias}

- Registros y gestión de activos (Records \& asset management - A.M.): incluye actividades como el inventario de la subestación, de la red y la planificación de la inversión en activos como estrategia de mantenimiento, la gestión del riesgo, apoyo a las decisiones y la asignación de presupuesto.

- Optimización y planificación operativa (Operational planning \& optimization P.O.): consiste en las funciones relativas a la simulación de operaciones de red, como la potencia de cálculo, simulación de flujos de conmutación, pronóstico del tiempo y el ímpetu de análisis de riesgos, etc. También la programación de interruptor de la acción y la programación de las importaciones de energía son parte de esta función de negocio.

- Mantenimiento y construcción (Maintenance \& construccion - M.C.): incluye todas las funciones teniendo en cuenta el mantenimiento y la inspección, la construcción, el diseño y planificación del trabajo. Algunos ejemplos de componentes abstractos son el cierre de órdenes de trabajo, estimación de costos de trabajo, gestión del flujo de trabajo, planificación de tareas de trabajo, los resultados de inspección sobre el terreno.

- Planificación de la extensión de la red (Network extencion planning - N.E.): considera los cálculos de la red, supervisión de la construcción y la gestión de su cumplimiento.

- Lectura del medidor y control (Meter reading \& control - M.R.): incluye la lectura de contadores y control de carga.

\section{Modelamiento del proceso del negocio OMS [7]}

A continuación se muestra el modelo para el proceso de negocio del OMS, mediante el cual se describe cómo se realiza el proceso de gestión de la distribución. Conjuntamente, se realiza los modelos de flujo de trabajo (Workflow), mediante los cuales se identifica quien o quienes realizan las actividades del proceso de gestión de de interrupciones. Para esto, se presenta los flujos de trabajo de las principales funciones de gestión de la red de distribución.

Para realizar el modelado del proceso de negocio se utilizará el diagrama de análisis, en tanto que para modelar los flujos de trabajo BPMN - Business Process Modelling Notation, (Notación para el Modelado de Proceso de Negocio), se empleará la herramienta Enterprise Architect 9.3.

Posteriormente se presentarán los modelos de flujo de trabajo, con respecto al procedimiento de interrupciones enfocándose a la Empresa Eléctrica Quito, para realizar un análisis de la situación actual de dicha empresa.

\subsection{Tipos de diagramas y notación a emplear}

Los modelos de proceso se pueden crear utilizando una notación gráfica que hace que sea más fácil ver las relaciones entre las actividades y seguir el flujo del proceso.

\section{Diagrama de análisis}

Un diagrama de análisis es una notación gráfica de actividades, más conocido como Lenguaje de Modelo Unificado (UML) simplificado, el cual es aplicado a los modelos de procesos de negocio y es utilizado para capturar procesos de negocio de alto nivel, que incluye sus características y necesidades esenciales. A continuación se hace una descripción de los elementos de la notación a utilizar:

Tabla 1. Notación de diagrama de análisis [7].

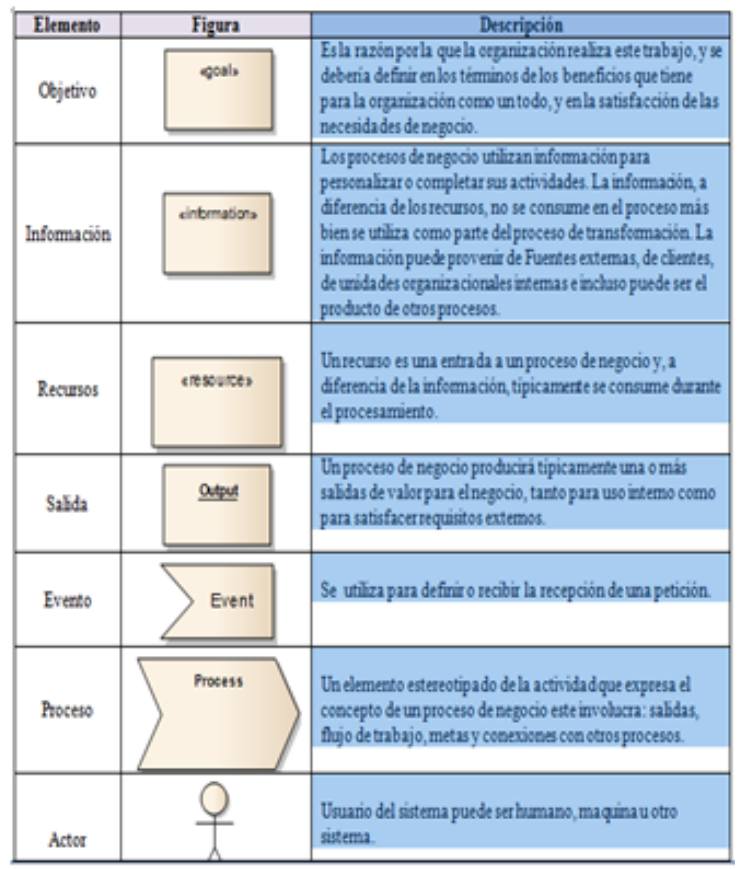

\subsubsection{Notación de modelos de procesos de negocio (BPMN)}

El modelamiento de procesos de negocio (Business Process Modelling -BPM), es una filosofía de gestión centrada en mejorar los procesos operacionales de la 
organización, y esta se apoya en BPMN que es la notación que facilita el modelamiento de procesos.

En este tipo de notación los procesos de negocio involucran la captura de una secuencia ordenada de actividades e información de apoyo.

\subsection{Descripción de la situación actual de la Empresa Eléctrica Quito, en base a su sistema de interrupciones de energía eléctrica}

La Empresa Eléctrica Quito es una empresa distribuidora de energía eléctrica, que cubre un área de concesión de $15000 \mathrm{~km}^{2}$, que corresponde al 5,85\% del territorio del Ecuador y comprende la provincia de Pichincha y parcialmente las provincias de Napo y Cotopaxi.

Actualmente, atiende a más de 870000 clientes con una participación en el mercado ecuatoriano cercana al $22 \%$ de la demanda nacional. La empresa cuenta con una capacidad instalada de 97 MW de generación hidráulica y 34,2 MW de generación térmica, además una potencia instalada superior a los 2,125 MVA a lo largo de 267,64 km de líneas de subtransmisión, 7 $348,88 \mathrm{~km}$. de redes de medio voltaje y $6588,85 \mathrm{~km}$ de redes de bajo voltaje que le permiten atender con altos estándares de calidad a sus clientes. La demanda es de 663,57 MW.

La Empresa Eléctrica Quito se encuentra en un proceso de actualización, desde inicios del 2009, en su sistema de control y comunicaciones, y por tanto, se obtuvo información relevante con las actualizaciones del sistema OMS de la EEQ.

\section{Análisis del proceso actual con el que se realiza el monitoreo y registro de las interrupciones de energía en la Empresa Eléctrica Quito [6] [8]}

Para realizar el análisis de las funciones relacionadas con la implementación de un OMS, se ha recopilado la información relacionada con las funciones y subfunciones principales del OMS, tales como las funciones que se describen en el numeral 2.1 de este trabajo, que son las funciones de negocio y las principales que se tratan son Customer Support y Network Operation, con la finalidad de detallar los beneficios y consecuencias de las interrupciones a realizarse por la Empresa Eléctrica Quito.
Tabla 2. Elementos principales empleados de la notación [7].

\begin{tabular}{|c|c|c|c|}
\hline \multicolumn{4}{|c|}{ 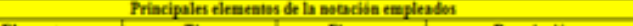 } \\
\hline Elemenato & Tipo & Figura & Descripciba \\
\hline Eventos & & & 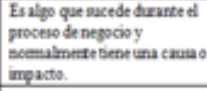 \\
\hline Evento & Inicio rimple & & 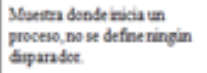 \\
\hline Evernts & lrátie por menaje & & 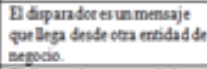 \\
\hline Evento & Inicio por sehal & & 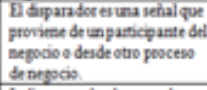 \\
\hline Everto & Inetemido & & 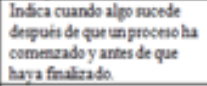 \\
\hline Everto & $\begin{array}{l}\text { Intemndo pet } \\
\text { tiempe }\end{array}$ & & $\begin{array}{l}\text { Ddippandor et una honay } \\
\text { fecha eqpetista ointeralo de } \\
\text { Dempe. }\end{array}$ \\
\hline Everto & Fnal & & $\begin{array}{l}\text { Marta cuado unprosete } \\
\text { frilien. }\end{array}$ \\
\hline Evento & Final per sehal & & 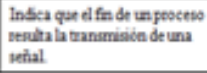 \\
\hline
\end{tabular}

\subsection{Análisis de la funciones del OMS a implementarse por la EEQ}

El proceso de la Automatización de la red por parte de la EEQ, está enmarcada en el proyecto $\mathrm{SIGDE}^{6}$, su eje estratégico es fortalecer la gestión de la operación y planificación operacional del sistema eléctrico de distribución con el fin de mejorar la calidad del servicio técnico, reducir el tiempo total de las interrupciones y su frecuencia, así como minimizar el tiempo de atención de reclamos, mejorar la planificación de la operación, eliminar la siniestralidad, entre otros. Para el análisis de los requerimientos de implementación para la EEQ se utiliza como referente internacional a la norma ICE 61968.

A continuación se detallan los resultados del análisis de cada una de las subfunciones obtenidas para cada función perteneciente a un OMS.

- Función de localización, aislamiento de fallas y restauración del servicio (FLIR)

La función FLIR está en la capacidad de utilizar toda la información disponible de telemetría del OMS, información de los dispositivos localizados en las subestaciones y a lo largo de los circuitos estado de interruptores, información del sistema AMI, etc. e información proveniente del sistema de atención de llamadas (TCS) y/o del sistema de gestión de eventos (OMS), por tanto, está en condiciones de manejar fallas trifásica, bifásicas, y monofásicas, de esta manera, se dispondrá de la capacidad de manejar múltiples fallas de distintos tipos, en diversos alimentadores.

\footnotetext{
${ }^{6}$ SIGDE: comité multidisciplinario conformado por funcionarios del MEER y de las Empresas Distribuidoras del Ecuador, con el objetivo de fortalecer los procesos de la operación de la red y de la planificación operacional de la gestión de las distribuidoras del país, con la finalidad de mejorar la calidad de servicio técnico, reducción del tiempo en atención a reclamos, mejorar la planificación de la operación, entre otros.
} 
- Sistema de atención de llamadas (TCS) El sistema OMS proporciona la función de atención de llamadas (TCS), la cual desde el Centro de llamadas (Call Center), registra y procesa la información de las llamadas de los clientes por interrupción del servicio eléctrico, determina las causas y ubicación de los eventos, ayuda a los operadores a restaurar eficientemente el servicio, a la vez que mantiene los registros detallados de las llamadas y suministra reportes significativos.

\subsection{Análisis de las normas internacionales para el intercambio de datos de la Empresa Eléctrica Quito [6] [8]}

Este artículo presenta las interfaces de sistema de gestión de distribución de la IEC, aspecto que promueve la Norma Internacional IEC 61968-1, que ha sido elaborada por el Grupo de Trabajo 14 del comité técnico IEC 57: "Control de energía del sistema y comunicaciones asociadas". IEC 61968 es una serie de normas, entre las más relevantes se puede citar a la Norma IEC 61968-3 Interface Standard For Network Operation (norma de interfaz para la operación de la red), ya que su información especifica las funciones de negocio relacionadas con la operación de la red.

Por lo tanto, la norma IEC 61968 es relevante para aplicaciones de acoplamiento flexible con una mayor heterogeneidad en los idiomas, los sistemas operativos, protocolos y herramientas de gestión. IEC 61968 está diseñado para soportar aplicaciones que se necesitan para intercambiar datos de forma controlada por eventos. IEC 61968 está diseñado para ser implementado con los servicios de middleware ${ }^{7}$, que se sitúa como intermediario de los mensajes entre las aplicaciones, también sirve como puertas de enlace de base de datos y tiendas operativas.

\subsection{Análisis de brechas existentes en la Empresa Eléctrica Quito [9]}

El análisis de las brechas existentes de la Empresa Eléctrica Quito, se centra exclusivamente en el estudio de las funciones de negocio presentadas por la norma internacional IEC, como son las N.O. Network Operation (Operación de la Red) y C.S. Customer Suport (atención al cliente).

\section{Función N.O.}

Según el plan estratégico de la EEQ, los objetivos a corto plazo en la función de N. O, que estima reducir el tiempo total de interrupciones, así como disminuir la duración individual de todas las interrupciones en el suministro de la electricidad al consumidor urbano.

\section{Función C. S.}

Los objetivos en atención al cliente, plantea ampliar el nivel de usuarios informados en forma individual previo a una interrupción programada, también mejorar índice de satisfacción en atención al cliente entre otros objetivos.

Como síntesis del análisis de brechas existentes en la Empresa Eléctrica Quito, se puede señalar que la implementación de los sistemas de Gestión de interrupciones OMS estarán tentativamente finalizadas para finales del año 2014, momento en el cual los sistemas SCADA/OMS-DMS del proyecto SIGDE entrarán en funcionamiento, a monitorear la red de distribución con las normas internacionales establecida por el IEC y el EPRI.

\subsection{Análisis costo beneficio para la implemen- tación de un OMS en la Empresa Eléctrica Quito [10]}

En esta sección se presenta el estudio económico del proyecto OMS, tomando como datos fundamentales el presupuesto referencial presentado para la oferta de implementación del SCADA/OMS, del proyecto del Sistema Integrado de Gestión de la Distribución Eléctrica "SIGDE" y el Comité de Gestión de la Operación del Sistema Eléctrico, quienes están encargados de la toma de decisiones sobre la adquisición de los componentes de software "OMS/DMS", orientados a mejorar la operación y planificación del sistema eléctrico de distribución, los cuales deben sujetarse a los lineamientos del MEER en la Adopción del modelo CIM (Common Information Model) definido en las normas IEC 61970/61968.

\subsection{Identificación, cuantificación valoración de ingresos, beneficios y costos [9]}

Los rubros de inversión como el de hardware, software, administración, ingeniería e implementación, capacitación y opcionales del Sistema, son datos obtenidos de la oferta presentada por la empresa TELEVENT ENERGÍA DEL ECUADOR para la implementación del OMS.

Las tasas de descuento financiero y económico correspondiente al valor del $12 \%$, que se regirá el presente análisis, son aquellas contempladas por la Secretaría Nacional de Planificación y Desarrollo (SENPLADES), las cuales son utilizadas en la evaluación económica de proyectos de inversión del Estado.

\footnotetext{
${ }^{7}$ Middleware es un software de computadora que conecta componentes de software o aplicaciones para que puedan intercambiar datos entre éstas. Es utilizado a menudo para soportar aplicaciones distribuidas. Esto incluye servidores web, servidores de aplicaciones, sistemas de gestión de contenidoy herramientas similares. Middleware es especialmente esencial para tecnologías como XML, SOAP, servicios web y arquitecturas orientada a servicios.
} 
Tabla 3. Rubros de inversión [11].

\begin{tabular}{lc}
\hline \multicolumn{2}{c}{ Rubros de inversión } \\
\hline Hardware & $3.684 .517,36$ \\
Software & $4.807 .801,27$ \\
Administración ingeniería e & \\
implantación & $4.891 .969,28$ \\
Capacitación & $80.824,85$ \\
\hline Opcionales del sistema & $123.470,30$ \\
Presupuesto total & $13.588 .583,06$ \\
\% Inversión EEQ & $3.193 .317,02$ \\
Tasa de descuento financiero & $12 \%$ \\
\hline Tasa de descuento económico & $12 \%$ \\
\hline
\end{tabular}

Hay que recalcar que el presupuesto referencial presentado para la oferta de implementación del SCADA/OMS, del proyecto del Sistema Integrado de Gestión de la Distribución Eléctrica "SIGDE" está contemplado para las empresas:

EEQ (Quito), EEASA (Ambato), Corporación Nacional de Electricidad S.A (CNEL), CENTROSUR (Cuenca), EEACA (Azogues), EERSSA (Loja) y EERSA (Riobamba), que suman un valor de TRECE MILLONES QUINIENTOS OCHENTA Y OCHO MIL QUINIENTOS OCHENTA Y TRES CON 06/100 USD.

Para realizar el análisis en relación a la Empresa Eléctrica Quito se utilizará el valor del presupuesto total por el porcentaje del $23,50 \%$ que corresponde al consumo nacional por áreas de concesión, presentado en el Plan Estratégico del CONELEC. Dándonos un valor de TRES MILLONES CIENTO NOVENTA Y TRES MIL TRESCIENTOS DIEZ Y SIETE MIL CON 02/100 USD. Que compete a la inversión del EEQ, el cual se utilizará para los cálculos de los índices económicos.

\subsection{Resultados}

\section{Análisis de resultados}

Tabla 4. Rubros de inversión [11].

\begin{tabular}{llc}
\hline \multicolumn{2}{c}{ Factibilidad } \\
\hline & \multicolumn{2}{c}{ Resultados } \\
& implementación OMS \\
\hline $\begin{array}{l}\text { Valor actual neto (VAN) } \\
\text { Tasa interna de retorno }\end{array}$ & 50.685 .728 & Viable \\
$\begin{array}{l}\text { Relación beneficio/costo } \\
\text { a valor presente (B/C) }\end{array}$ & 2,30 & Viable \\
$\begin{array}{l}\text { Factor de recuperación } \\
\text { de capital (FRC) }\end{array}$ & Beneficioso \\
$\begin{array}{l}\text { Costo equivalente anual } \\
\text { (CEA) }\end{array}$ & \\
\hline
\end{tabular}

Con los datos recopilados en las tablas anteriores, podemos obtener el resultado de la factibilidad de este proyecto, teniendo como base 5 años. El factor de recuperación de capital (FRC) es de 0,88 años, en el cual el retorno de inversión (RI), es de 20.106.330,73 USD de utilidad para la EEQ, esto en relación al valor de $23.2999 .647,75$

\section{Conclusiones}

En el estudio de los sistemas de interrupciones se utiliza las normas internacionales de institutos reconocidos a nivel mundial en la elaboración de estándares para los Smart Grid como son el EPRI y el IEC.

Las funciones principales en el análisis de las interrupciones se basan en la norma IEC, que son las operaciones de la red, y atención al cliente.

Las brechas existentes en la Empresa Eléctrica Quito se minimizarán con la implementación del sistema OMS, logrando alcanzar, así las metas a corto plazo deseadas.

\section{Referencias}

[1] A. Espinosa, S. González, and B. Sierra, "Automatización de la distribución: presente y futuro," Revista Divulgación, pp. 4752, Junio 2011. [Online]. Available: http: //www.iie.org.mx/boletin022011/divulga.pdf

[2] SOLUS Ingeniería de Software, "El lenguaje común de las smart grids: Unified modeling lenguage," Publicado por Solus, Mendoza, Argentina, 2010.

[3] "Plan Maestro de Electrificación del Ecuador 2012-2021," Ministerio de Electricidad y Energía Renovable, CONELEC, CELEC, corporación CENACE, Tech. Rep., 2012.

[4] EEQ, "Índices de Gestión 2000 - junio 2012," Revista Gestión, pp. 15-17, 2012.

[5] NIST, "Frame word and roadmap for smart grid. interoperability standards, release 1.0," Office of the National Coordinator for Smart Grid Interoperability, January 2012, NIST Special Publication 1108.

[6] "IEC-61968-1 Interface Architecture and General Requirements," Ginebra, Suiza, 2002.

[7] K. Chuluisa and L. Martínez, "Estudio de los sistemas para la administración de la distribución DMS y de los requerimientos para su implementación," Proyecto de Graduación, Universidad Politécnica Salesiana, Ecuador, Diciembre 2011.

[8] EEQ, "Especificaciones Técnicas del sistema SCADA/OMS-MWM/DMS," Proyecto SIGDE, Quito, Tech. Rep., Agosto 2012. 
[9] ——, Guía Estratégica EEQ 2011 - 2015, versión enero 2012 ed., Empresa Eléctrica Quito, Quito.

[10] ——, "Resolución de gerencia," GEG, 18-18-2012.
[11] "Oferta presentada para la implementación OMS/DMS para la EEQ," Propiedad de TELEVENT ENERGÍA DEL ECUADOR, 2012. 